\title{
Hemorrhagic Cholecystitis in a Patient with Cirrhosis and Rectal Cancer
}

\author{
Rony Shah ${ }^{1}$, Linda C. Klumpp ${ }^{1}$, James Craig ${ }^{1}$, Parth Patel ${ }^{2}$, Jeffrey Jordan ${ }^{1}$ \\ 1. Internal Medicine, Citrus Memorial Hospital, Inverness, USA 2. Surgery, Citrus Memorial Hospital, Inverness, USA
}

Corresponding author: Rony Shah, rony.shah@hcahealthcare.com

\begin{abstract}
Hemorrhagic cholecystitis is a rare presentation of acute calculous cholecystitis which presents with abdominal pain, jaundice, and gastrointestinal bleeding. It is a challenging diagnosis to make because it present similar to other common disorders such as calculous cholecystitis. We present a unique case of hemorrhagic cholecystitis in a patient with cirrhosis and rectal cancer.

A 66-year-old male with a history of rectal cancer, alcohol-induced cirrhosis, esophageal varices, stroke, paroxysmal atrial fibrillation, and hypertension presented to the emergency department with complaints of abdominal pain. Patient's computed tomography (CT) scan revealed bleeding from the gallbladder with hemoperitoneum and thickening of the ascending colon. The patient underwent emergent surgery for hemorrhagic cholecystitis.
\end{abstract}

Hemorrhagic cholecystitis is associated with risk factors, including trauma, malignancy, renal failure, cirrhosis, and anticoagulation therapy. Imaging is not always reliable, but ultrasound and CT scan are the preferred options. Treatment options are surgical or nonsurgical approach depending on patient's hemodynamic stability.

Categories: Internal Medicine, Gastroenterology, General Surgery

Keywords: cholecystitis, hemorrhagic cholecystitis, hemoperitoneum, cirrhosis, rectal cancer

\section{Introduction}

Hemorrhagic cholecystitis is a life-threatening complication of acute calculous cholecystitis. A hemorrhage within the gallbladder can occur for a variety of reasons, such as obstructive cholecystitis, biliary neoplasm, biliary parasites, bleeding disorder, percutaneous intervention, and trauma [1]. It is a challenging diagnosis to make because its presentation is similar to calculous cholecystitis. Hemorrhagic cholecystitis typically presents with abdominal pain, jaundice, and gastrointestinal bleeding which is found in $22 \%$ of cases [2]. We present a unique case of hemorrhagic cholecystitis in a patient with cirrhosis and rectal cancer.

Received 03/23/2020

Review began 03/25/2020 Review ended 04/21/2020 Published 04/29/2020

\section{() Copyright 2020}

Shah et al. This is an open access article distributed under the terms of the Creative Commons Attribution License CC-BY 4.0., which permits unrestricted use, distribution, and reproduction in any medium, provided the original author and source are credited.

\section{Case Presentation}

A 66-year-old male presented to the emergency department (ED) with complaints of abdominal pain. The patient provided a past medical history of rectal cancer, alcohol-induced cirrhosis, esophageal varices, stroke, paroxysmal atrial fibrillation, and hypertension. Vital signs at admission were temperature 99 degree Fahrenheit, heart rate 111 beats per minute, respiratory rate 19 blood breaths per minute, pressure 132/82 $\mathrm{mmHg}$, and oxygen saturation 95\%. Physical examination was positive for right upper quadrant tenderness. Laboratory results showed white blood count $14,000 / \mathrm{mm}^{3}$, hemoglobin $9.5 \mathrm{~g} / \mathrm{dL}$, platelet count $77 \times 10^{9} / \mathrm{L}$, aspartate aminotransferase $19 \mathrm{U} / \mathrm{L}$, alanine transaminase $12 \mathrm{U} / \mathrm{L}$, alkaline phosphatase $85 \mathrm{U} / \mathrm{L}$, prothrombin time/international normalized ratio 36/1.3. A CT of the abdomen and pelvis without contrast revealed cirrhotic liver, cholelithiasis, and a mass extending to the gallbladder from the medial segment of the left hepatic lobe (Figure 1). 


\section{Cureus}

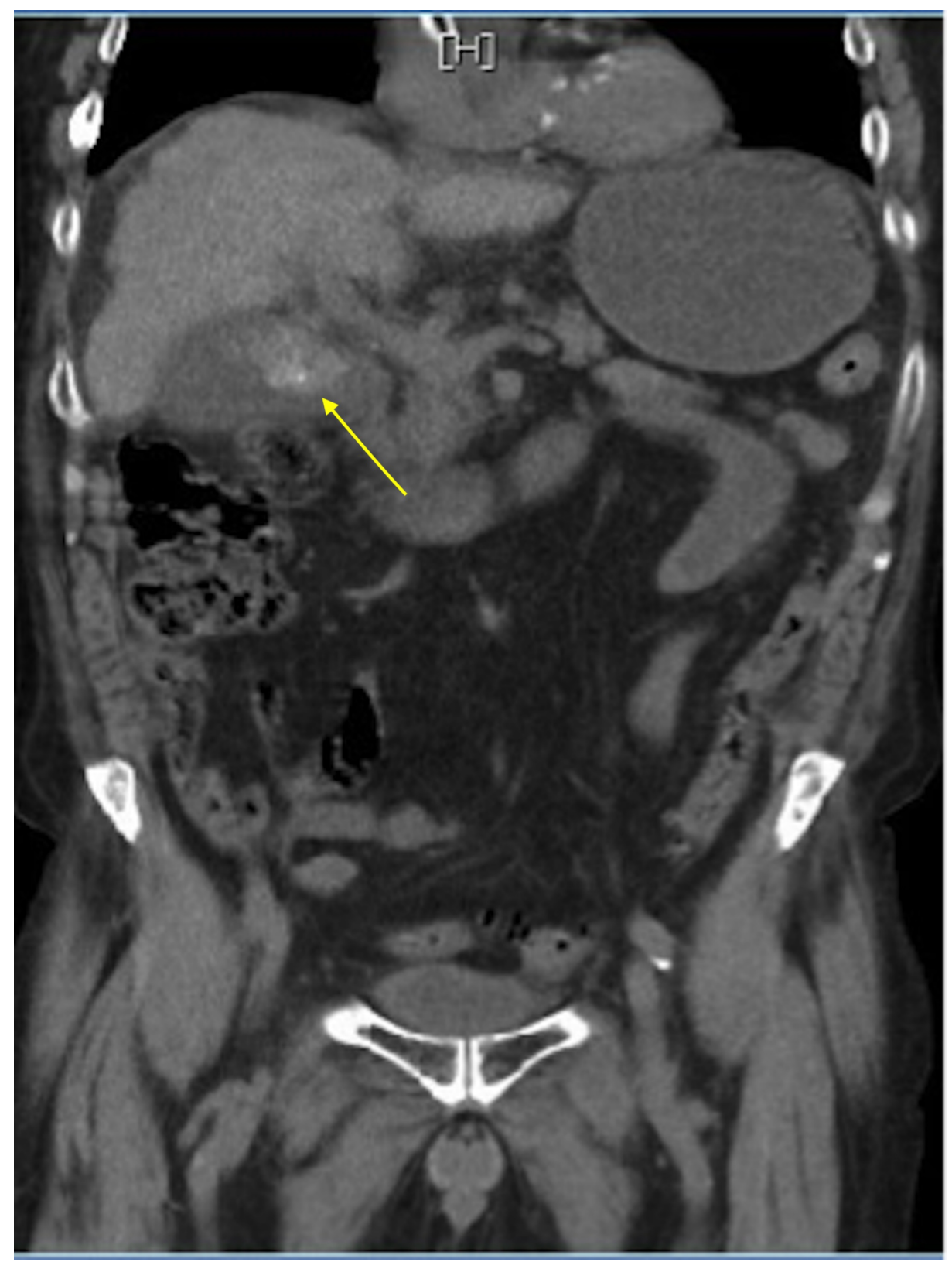

\section{FIGURE 1: CT of the abdomen and pelvis without contrast}

Cholelithiasis with enlarged gallbladder or liver mass

Ultrasound of the right upper quadrant revealed cholelithiasis with a distended gallbladder. The patient reported he had been recently treated with neoadjuvant chemotherapy and radiation for rectal cancer. His positron emission tomography/CT scan and MRI were negative one month prior to his ED visit. He developed multifocal atrial tachycardia and later wide QRS tachycardia soon after admission. The irregular rhythm was managed with beta-blocker therapy, oral metoprolol $50 \mathrm{mg}$ twice a day. The patient had one episode of hematemesis and developed shock requiring aggressive intravenous fluid resuscitation and vasopressors. Rapid sequence intubation was required for acute hypoxemic respiratory failure due to possible aspiration. Bronchoscopy was performed afterwards and dark brownish secretions were suctioned. A repeat $\mathrm{CT}$ of the abdomen and pelvis without contrast showed bleeding from the gallbladder with hemoperitoneum and thickening of the ascending colon (Figure 2). The patient was emergently taken to surgery and underwent laparoscopic cholecystectomy with evacuation and drainage of intra-abdominal hematoma/hemoperitoneum and abscess. During surgery, a large hemoperitoneum was found throughout the abdomen, and after evacuating $1.5 \mathrm{~L}$ of cold blood and bile, the gallbladder was visualized. Bright red blood was oozing from the cystic artery, and the gallbladder was perforated with leakage of bile and blood throughout the abdomen. Active bleeding from the cystic artery was stopped with a staple because artery dissection and placement of a clip were not possible. The patient had two drains placed intra-abdominally, and his gallbladder was sent for pathology. He remained hemodynamically stable and was extubated four 


\section{Cureus}

days later. Gallbladder pathology did not show any evidence of malignancy. Intra-abdominal cultures were positive for Klebsiella pneumoniae and Enterobacter cloacae due to gallbladder perforation. The patient was treated with appropriate antimicrobial therapy (pipercillin/tazobactam and meropenem) per culture sensitivity for seven days. He had an outpatient follow-up with the general surgeon in two weeks, and no complications were noted.

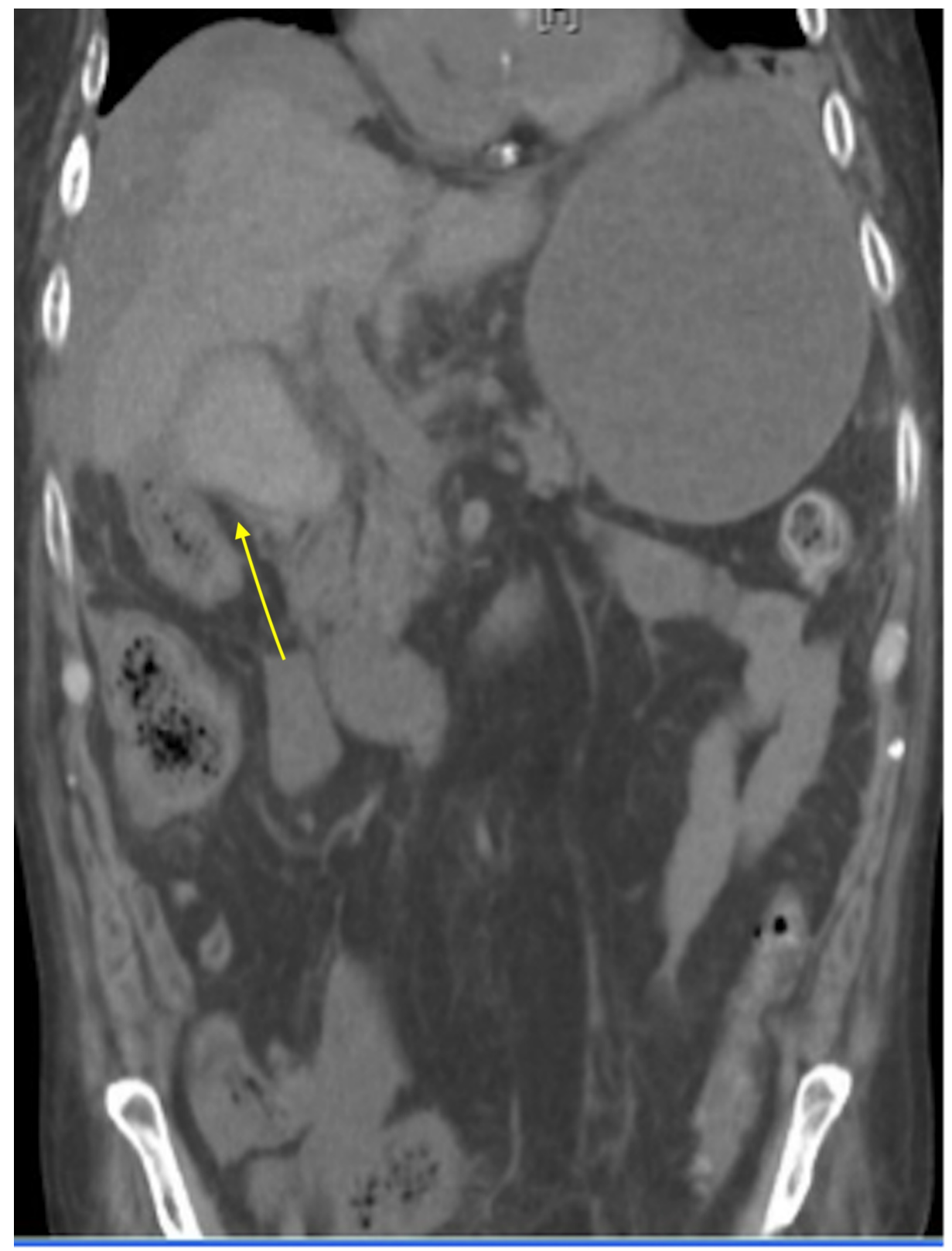

FIGURE 2: CT of the abdomen and pelvis without contrast

Hemorrhage within the gallbladder resulting in a subcapsular hematoma

\section{Discussion}

In 1979, Shah and Clegg described hemobilia caused by cholecystitis as hemorrhagic cholecystitis $[3,4]$. Hemorrhagic cholecystitis can be difficult to diagnosis because it presents similarly to acute calculous cholecystitis and imaging is unreliable. Associated risk factors include trauma, malignancy, renal failure, cirrhosis, and anticoagulation therapy [5]. In our case, the patient had rectal cancer and history of cirrhosis. Cholelithiasis-related microbleeding of the gallbladder accounts for $9 \%$ of the hemobilia cases [3].

Hemorrhagic cholecystitis occurs due to transmural inflammation of the gallbladder wall leading to ischemia and mucosal breakdown, erosion into gallbladder vessels, and hemorrhage into the gallbladder lumen or abdominal cavity [6]. In majority of the reported cases, common presentation is right upper quadrant pain associated with fever and leukocytosis. Complications such as peritonitis, hematemesis, and melena may occur [6]. Hemorrhagic cholecystitis is associated with high mortality and morbidity, especially with 
gallbladder perforation and massive hemorrhage [7]. The mortality and morbidity rates associated with gallbladder perforation range between $15 \%-20 \%$ and $32 \%-58 \%$, respectively [8]. Our patient presented with abdominal pain, leukocytosis, hematemesis, and gallbladder perforation leading to hemoperitoneum. Abdominal ultrasound is the investigation of choice for diagnosing upper-right quadrant pathology. Ultrasound findings typically show gallbladder wall thickening, intraluminal membranes, and nonshadowing, nonmobile intraluminal echogenic material $[2,9]$. A previous study by Chinn et al. revealed that $74 \%$ of patients reviewed had similar ultrasound findings [6]. CT findings typically show contrast extravasation during the arterial phase, high attenuation within the gallbladder lumen and fluid-fluid layering $[2,10]$. Image findings may be able to distinguish acute calculous cholecystitis from hemorrhagic cholecystitis. In our case, the repeat CT showed bleeding from the gallbladder with hemoperitoneum. Treatment option for hemorrhagic cholecystitis is either cholecystectomy or cholecystostomy, which is preferred in patients too hemodynamically unstable for surgery. Our patient underwent laparoscopic cholecystectomy with evacuation of intra-abdominal hemoperitoneum and abscess. Although he required intensive perioperative care, he was fortunate enough to make a complete recovery. Hemorrhagic cholecystitis is a rare complication that needs to be considered in high-risk patients.

\section{Conclusions}

Hemorrhagic cholecystitis is associated with a high mortality and morbidity due to its life-threatening complications. Hemorrhagic cholecystitis can be difficult to diagnose; however, if clinically suspected, it warrants prompt medical investigation. Physician awareness with early identification and treatment can help prevent complications and death.

\section{Additional Information \\ Disclosures}

Human subjects: Consent was obtained by all participants in this study. Conflicts of interest: In compliance with the ICMJE uniform disclosure form, all authors declare the following: Payment/services info: All authors have declared that no financial support was received from any organization for the submitted work. Financial relationships: All authors have declared that they have no financial relationships at present or within the previous three years with any organizations that might have an interest in the submitted work. Other relationships: All authors have declared that there are no other relationships or activities that could appear to have influenced the submitted work.

\section{Acknowledgements}

This research was supported in part by HCA Healthcare and/or an HCA Healthcare affiliated entity. The views expressed in this publication represent those of the author(s) and do not necessarily represent the official views of HCA Healthcare or any of its affiliated entities.

\section{References}

1. Kwon J: Hemorrhagic cholecystitis: report of a case . Korean J Hepatobiliary Pancreat Surg. 2012, 16:120122. 10.14701/kjhbps.2012.16.3.120

2. Sweeny A, Smith N, Serfin J: Hemorrhagic cholecystitis causing hemobilia and common bile duct obstruction. J Surg Case Rep. 2019, 2019:rjz081. 10.1093/jscr/rjz081

3. Shishida M, Ikeda M, Karakuchi N, et al.: Hemorrhagic cholecystitis in a patient on maintenance dialysis . Case Rep Gastroenterol. 2017, 11:488-493. 10.1159/000479497

4. Shah VR, Clegg JF: Haemorrhagic cholecystitis. Br J Surg. 1979, 66:404-405. 10.1002/bjs.1800660608

5. Parekh J, Corvera C: Hemorrhagic cholecystitis. Arch Surg. 2010, 145:202-204. 10.1001/archsurg.2009.265

6. Morris DS, Porterfield JR, Sawyer MD: Hemorrhagic cholecystitis in an elderly patient taking aspirin and cilostazol. Case Rep Gastroenterol. 2008, 2:203-207. 10.1159/000135693

7. Donn E, Atkinson I, McCague A: Hemorrhagic cholecystitis after warfarin use for deep vein thrombosis . Surg J. 2018, 4:e102-e104. 10.1055/s-0038-1660450

8. Kolder D, Geiger T, Tharakan AK, Kessel JW, Awad ZT: Massive hemoperitoneum from transhepatic perforation of the gallbladder. Mt Sinai J Med. 2006, 73:1135-1136.

9. Chinn DH, Miller El, Piper N: Hemorrhagic cholecystitis: sonographic appearance and clinical presentation . J Ultrasound Med. 1987, 6:313-317. 10.7863/jum.1987.6.6.313

10. Pandya R., O’Malley C: Hemorrhagic cholecystitis as a complication of anticoagulant therapy: role of CT in its diagnosis. Abdom Imaging. 2008, 33:652-653. DOI: 10.1007/s00261-007-9358-2 